\title{
Passivation of silicon surface by ultrathin dielectric film in M/Si/nematic/ITO structures
}

\author{
M.I. Gritsenko, S.I. Kucheev, P.M. Lytvyn* \\ Chernigov Pedagogical University, 53, G.Polubotka str., 14038 Chernigov, Ukraine \\ *V. Lashkaryov Institute of Semiconductor Physics, prospect Nauky 45, 03028 Kyiv, Ukraine \\ E-mail:kucheev@cn.relc.com
}

\begin{abstract}
In this work, the experimental observation of passivation of silicon surface by ultrathin dielectric film in $\mathrm{Al} / \mathrm{Si} /$ nematic/Indium Tin Oxide(ITO) structure performed on the base of low resistive ( $\sim$ several $\Omega \times \mathrm{cm}) n$-type conductivity silicon sample is presented. It is shown that when the DC voltage with polarity $+U$ on silicon is applied to the structure a passivation effect of silicon surface takes place. It is found that a silicon surface passivated by dielectric film changes an initial alignment of liquid crystal 5CB. In the structure with passivating silicon surface, a frequency range of structure photosensitivity extends due to limitation of current leakage through Si/liquid crystal interface.
\end{abstract}

Keywords: nematic, silicon, passivation, alignment, photosensitivity, light modulator.

Paper received 15.12.03; accepted for publication 17.06.04.

\section{Introduction}

Metal $/ \mathrm{Si} / \mathrm{SiO}_{2} /$ nematic/transparent electrode structures $\left(\mathrm{M} / \mathrm{Si} / \mathrm{SiO}_{2} /\right.$ nematic/ITO) are used in a broad range of electrooptical devices including optically addressed space light modulators (OA SLM) [1-4]. A functional operation of these structure is based on a silicon surface depletion effect [5] that is caused by means of external electric field of ITO electrode. $\mathrm{SiO}_{2}$ oxide film having thickness up to $0.1-1 \mu \mathrm{m}$ possesses a well insulating properties and frequently playing a role of dielectric light mirror. Such dielectric films fully exclude a charge transferring from a field electrode into silicon sample. A controlled charge embedded into oxide and repeatability of properties of $\mathrm{Si}-\mathrm{SiO}_{2}$ interface ensure a high stability of such devices [6].

However, the structures containing no $\mathrm{SiO}_{2}$ dielectric film, i.e. $\mathrm{M} / \mathrm{Si} /$ nematic/ITO structures, possess more extended functional possibilities because, in this case, a surface conductivity of silicon can be additionally modulated: 1) by electric field of ion cloud, which can be localized very close to silicon surface and 2) by means of current leakage through Si/LC interface. Such structures being exploited as OA SLMs, for example, can separate moving and stop images [7]. Such type of structures allows to easily realize an ion "quasi" electrode conception when the locally accumulated charge within liquid crystal layer plays a role of the field electrode [7]. Using a field effect in silicon induced by ion charges [8], there is a possibility to study the ion processes taking place in liquid crystals.

Meantime, the charges flowing through $\mathrm{Si} / \mathrm{LC}$ interface can cause the modification of silicon surface. One of the most probable process could be gone on silicon surface under the action of DC (as well AC) voltage is a passivation of silicon surface by dielectric film.

The aim of the present work was to reveal a passivation effect on silicon surface in a prototype of liquid crystal device under the action of DC voltage. In the work, several independent methods were used to confirm a passivation effect.

\section{Experimental and results}

The structure performed on the base of n-type conductivity monocrystalline silicon (see Fig.1) and specific resistivity of $4.5 \Omega \times \mathrm{cm}$ was used in order to reveal the passivation effect of silicon surface in $\mathrm{M} / \mathrm{Si} /$ nematic/ITO structures. The upper electrodes were performed as ITO stripes. Before assembling the structure the silicon surface was etched in HF solution and then rinsed in distillate water. Liquid crystal 5CB layer thickness was $20 \mu \mathrm{m}$. After this the $\mathrm{DC}$ voltage equal to $4.4 \mathrm{~V}$ with polarity $+U$ 


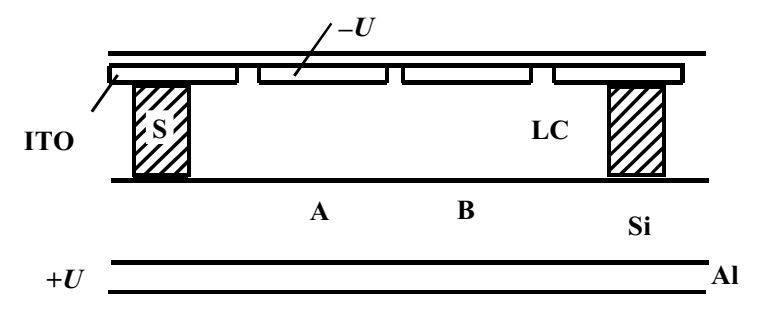

Fig. 1. The cross-section of investigated $\mathrm{A} 1 / \mathrm{Si} / 5 \mathrm{CB} / \mathrm{ITO}$ structure. $S$ denotes a spacer.

on silicon substrate was applied to structure for 5 minutes. The current density was equal to $\sim 1 \mu \mathrm{A} / \mathrm{cm}^{2}$. Then the structure was disassembled, and the silicon surface is cleaned only by organic dissolvent: dimetilformamide and hexane (so $\mathrm{SiO}_{2}$ was not etched).

The investigation of silicon surface with optical microscope showed that both "A" and "B" silicon surfaces that lie opposite the stripes (Fig.1) are optically identical. ("A" and "B" surfaces lie opposite the stripes on which the voltage $U$ was applied and not applied, respectively).

The "A" and "B" surfaces are well detected by means of a water dew condensation and evaporation. From " $A$ " surface a dew evaporates slower than from "B" surface, what is well seen by the naked eye. This means that the roughness of " $A$ " surface is large than roughness of " $B$ " surface. Taking into account that " $A$ " and " $B$ " surfaces are optically identical, the difference between their roughness must be at a nanoscale level.

" $A$ " and "B" surfaces were examined by the atomic force microscope (AFM, Digital Instruments NanoScope IIIa in tapping mode) in scanning areas of $1 \times 1 \mu \mathrm{m}$. The surface topography in points "A" and "B" is shown as height maps in Fig. 2. As it is well seen, the morphology of these fragments of "A" and "B" surfaces are different. On "A" surface the round pores (Fig.2a, black small spots) are observed. A typical profile of single pore on "A" surface is presented in Fig. $2 c$. The depth of pore is approximately equal to several nanometers and its diameter is 40 nanometers, respectively. We suppose that the pores have been appeared on "A" surface by the following way. It is well known [6] that in the course of anode oxidizing the silicon surface contacting with electrolyte, silicon oxide film grows. Varying electrolyte mixture the oxide film having required porosity can be obtained. In our case a liquid crystal 5CB plays a role of weak electrolyte [9]. Therefore, when an external voltage with $+U$ polarity on silicon is applied to $\mathrm{Al} / \mathrm{Si} / 5 \mathrm{CB} / \mathrm{ITO}$ structure, a dielectric film grows on silicon surface. We believe that this dielectric film is silicon oxide. Taking into account that the radius of AFM tip is equal to $\sim 5 \ldots 10 \mathrm{~nm}$, we assume also that the minimal value of silicon oxide film thickness is equal approximately to depth of a pore, i.e. $3 \ldots 4 \mathrm{~nm}$.

It should be noted from the results of AFM examination of silicon surface it is followed that both " $A$ " and "B" surfaces are clean, i.e. there are no any contamina-
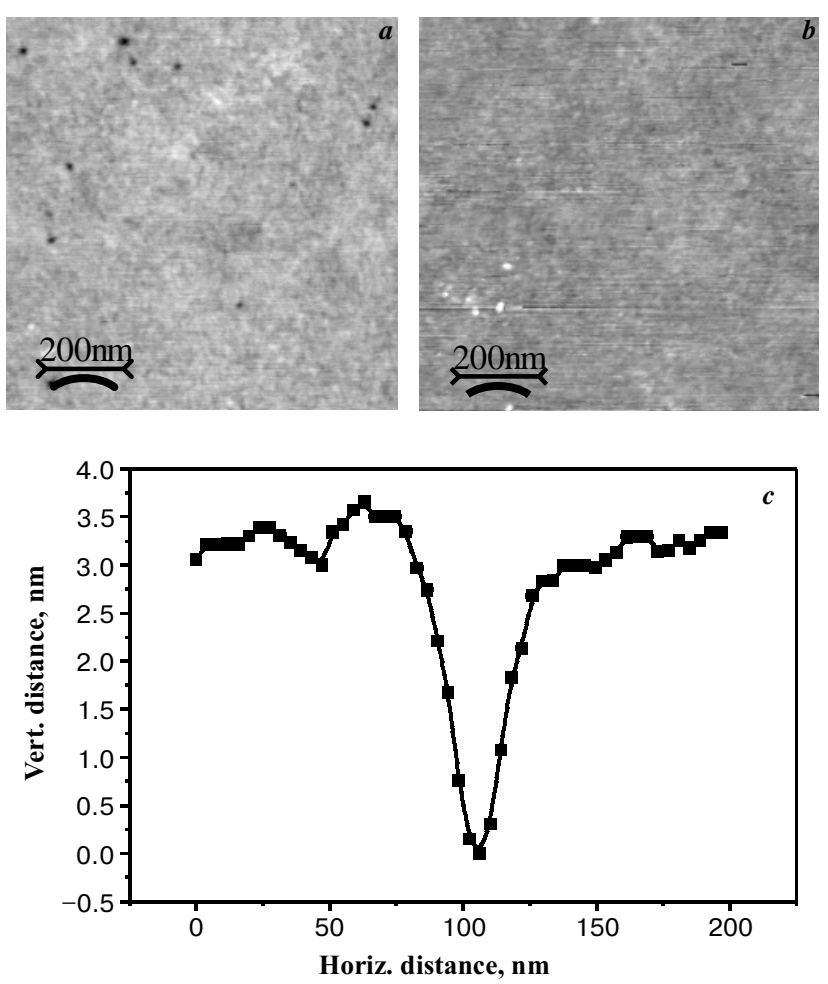

Fig. 2. AFM images of Si surface in points "A" $(a)$ and "B" $(b)$ shown in Fig. 1 after removing of liquid crystal; $(c)$ - the profile of pore on surface $(a)$.

tion which could influence on the alignment of liquid crystals in the following experiments.

On the base of above investigated silicon sample containing " $A$ " and " $\mathrm{B}$ " surfaces the $\mathrm{Al} / \mathrm{Si} / 5 \mathrm{CB} / \mathrm{ITO}$ structure was assembled in the way where the upper ITO electrode was unbroken. In order to obtain perfect homogeneous planar alignment of $5 \mathrm{CB}$ liquid crystal on whole surface of ITO electrode, the surface was covered by rubbed polyimide film. Liquid crystal thickness was $20 \mu \mathrm{m}$. Fig. 3 shows the fragment of A1/Si/5CB/ITO struc-

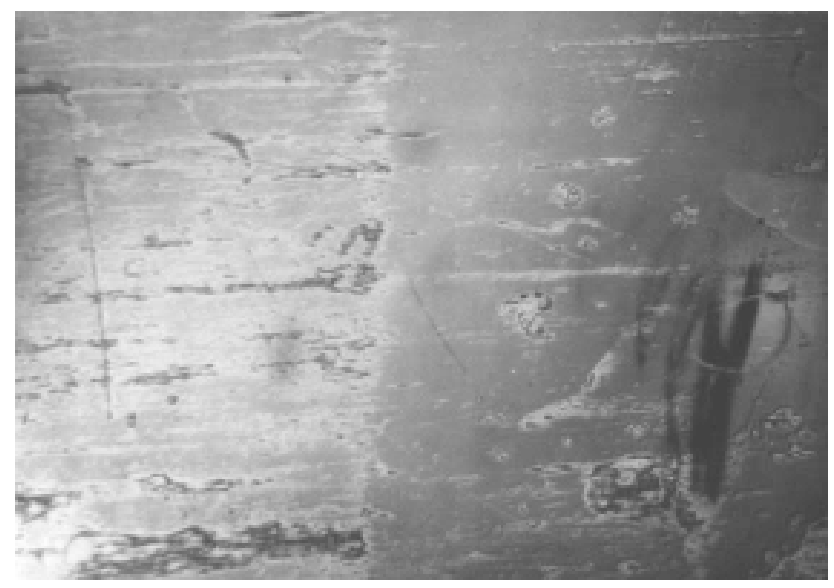

Fig. 3. Image of " $A$ " and " $B$ " fragments of silicon surface in $\mathrm{Al} / \mathrm{Si} / 5 \mathrm{CB} / \mathrm{ITO}$ structure with polarized light. 
M.I. Gritsenko et al.: Passivation of silicon surface by ultrathin dielectric film ...

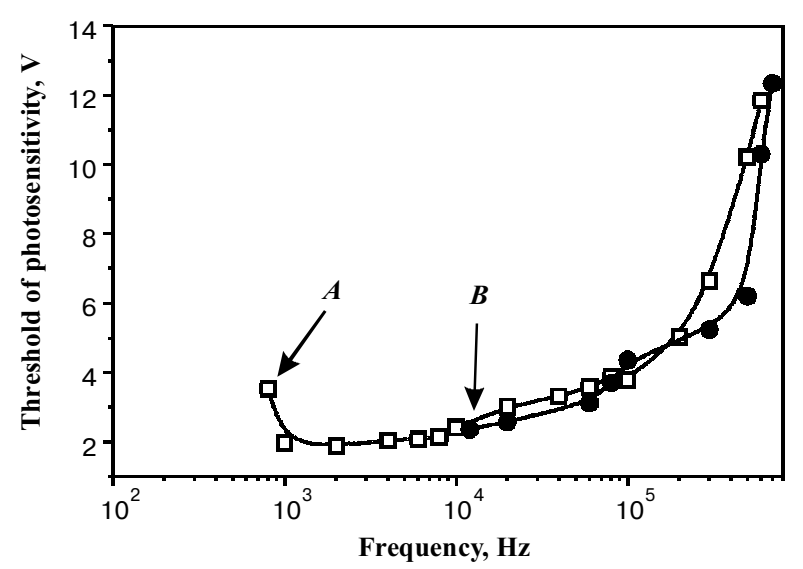

Fig. 4. Frequency dependence of threshold voltage applied to $\mathrm{Al} / \mathrm{Si} / 5 \mathrm{CB} / \mathrm{ITO}$ when a structure photo-sensitivity appears. Illumination by He-Ne laser $(0.63 \mu \mathrm{m}, P \sim 2 \mathrm{~mW})$.

ture with silicon surface includes "A" and "B" regions. As seen, a liquid crystal layer well decorates " $\mathrm{A}$ " and "B" surfaces, respectively. It means that there takes place the difference of liquid crystal alignment on " $\mathrm{A}$ " and " $\mathrm{B}$ " surfaces (taking into account that the molecules are homogeneously oriented on upper ITO electrode).

Then we studied a photosensitivity of the $\mathrm{Al} / \mathrm{Si} / 5 \mathrm{CB} /$ ITO structure in " $\mathrm{A}$ " and " $\mathrm{B}$ " areas. The photosensitivity of structure is determined by the reaction (yes/no) of liquid crystal on He-Ne laser illumination of corresponding area of silicon surface. When external AC voltage is applied to $\mathrm{Al} / \mathrm{Si} /$ nematic/ITO structure the surface depletion layer in silicon may be produced and almost whole voltage drops across depleted layer. Within a laser spot a liquid crystal reorients because depleted layer in silicon is not formed and the total voltage applied to structure drops across liquid the crystal layer. In the case of $n$ type silicon, a depletion of silicon surface takes place when the polarity of AC voltage is $+U$ on silicon. Because of a liquid crystal layer, in general case, is not isolated by dielectric film, a surface density of major carriers in silicon depends strongly on the current leakage $J$ through $\mathrm{Si} / \mathrm{LC}$ interface. In order to obtain a silicon surface depletion (in other words a structure photosensitivity), it is required that the major carriers flow from surface into bulk induced by external electric field must be larger than the total current consisting of the current leakage $J$ and the current due to thermo- and light-generated non-equilibrium carriers. Obviously, the ultrathin dielec- tric film on silicon surface can influence on value of current leakage $J$ and therefore on the structure photosensitivity.

Figure 4 shows the dependence of the threshold voltage of structure photosensitivity appearance on the frequency of the applied voltage. As seen, the frequency of structure photosensitivity appearance in "A" area (arrow on A curve) is more than an order of magnitude lower than the frequency of structure photosensitivity appearance in "B" area (arrow on B curve). It means that the current leakage $J$ through Si/LC interface in "A" surface of silicon is limited by a dielectric film grown on silicon surface.

\section{Conclusions}

In summary, we have found a passivation effect in the liquid crystal structure as a prototype of OA SLM. When the external voltage with polarity $+U$ on $\mathrm{Si}$ is applied to $\mathrm{Al} / \mathrm{Si} / 5 \mathrm{CB} / \mathrm{ITO}$ structure a silicon surface, prior etched in HF solution, is passivated by an ultrathin dielectric film. The passivating film changes an initial alignment of liquid crystal and limits the transfer of carriers through $\mathrm{Si} / \mathrm{LC}$ interface that promotes the formation of depleted surface layer in silicon under the action of AC voltage within more wide range of frequency.

\section{References}

1. J.D. Margerum, L.J. Miller, Electro-optical applications of liquid crystals // J. Colloid and Interface Science, 58(3), pp.559580 (1977).

2. D.G. Sikharulidse, G.S. Chilaya. Images transducers of MOSelectrooptic material type. "Radio i svyaz" Moscow (1986) (in Russian).

3. N.F. Kovtonyuk, E.N. Sal'nikov Photosensitive MOS-devices for image transducers. "Radio i svyaz"” Moscow (1990) (in Russian).

4. A.A. Vasiliev et al., // Optic, 67(3), pp. 223-236(1984).

5. R. Muller, T. Kamins. Device Electronics for Integrated Circuits, Wiley (1986).

6. R.M. Burger, R.P. Donovan. Oxidation, diffusion and epitaxy. Fundamentals of silicon integrated device technology. V.I "Mir" Moscow (1969) (in Russian).

7. M.I. Gritsenko, S.I. Kucheev, Ion controlled liquid crystal spatial light modulator // Proc.of SPIE, 5257, pp.145-151 (2003).

8. M.I. Gritsenko, S.I. Kucheev, Ion induced field effect in silicon in nematic liquid crystal cell // Semicond. Phys., Quant. Elect. and Optoelect., 6(2), pp.129-133 (2003).

9. H. Yokoyama, Handbook of Liquid Crystal Research, Oxford Press, New York (1997). 